\title{
Prediction of the comparative intensity of pneumoconiotic changes caused by chronic inhalation exposure to dusts of different cytotoxicity by means of a mathematical model
}

\author{
B A Katsnelson, L K Konyscheva, N Ye Sharapova, L I Privalova
}

\begin{abstract}
A multicompartmental mathematical model has been used to simulate variations in the cytotoxicity of dusts in the kinetics of the retention, in the pulmonary region and tracheobronchial lymph nodes, of practically insoluble quartzite and titanium dioxide dust particles deposited on the free surfaces of the acini from alveolar air. Experiments with these dusts were conducted on rats exposed to virtually the same dust concentrations in the air for an experimental period of 20 weeks and a period of 10 weeks after exposure. Satisfactory approximation to the experimental data on the retention of these dusts is obtained by using the model parameters that depend either on damage to lung macrophages by phagocytosed particles or on the response of the host organism to this damage by enhanced recruitment of neutrophilic leucocytes; all the other variables of the model being unchanged. The values of the "action integral" computed from this model and multiplied by the index of comparative cytotoxicity of particles in vitro satisfactorily approximate to quantitative differences in the intensity of pneumoconioses caused by the dusts under study by the end of the experimental period. On the whole, the results of the mathematical model agree with the hypothesis that the cytotoxicity of particles plays a key part in both the process of retention of dust in the lung parenchyma and lung associated lymph nodes, and the pathological process caused by the retained dust. Thus given the factors and conditions on which the deposition of practically insoluble dusts in the pulmonary region depends, it is necessary to take into account the multiplicative nature of these two effects of cytotoxicity when predicting the comparative risk of pneumoconiosis.
\end{abstract}

(Occup Environ Med 1994;51:173-180)

The breakdown of lung macrophages as a result of ingesting poorly soluble dust particles is a key link in the pathogenesis of pneumoconioses. This link determines both the effectiveness of lung clearance (and thus the kinetics of retention of dust in the lung parenchyma and lung associated lymph nodes) and the intensity of pathological changes in these organs caused by long term dust retention. Most of the researchers in this field share this view, with subtle differences. It still remains, however, a hypothesis that has not been treated quantitatively. The dual effect of cytotoxicity of dust is not disputed, but the statement that it has a key role still remains a speculative one.

One of the aspects of this problem may be formulated as the question: will the differences in the cytotoxicity of two practically insoluble mineral dusts be sufficient to explain experimental quantitative differences in both the kinetics of their retention and the intensity of the pneumoconiotic processes caused by them, provided that the conditions controlling the deposition of their particles in the pulmonary region of the lung are similar? This question is important both theoretically and practically. A positive answer to it would be a strong argument in favour of the hypothesis under study) and would ensure more reliable prediction of the comparative risk of pneumoconiosis due to dusts, the cytotoxicity of which was compared in short term tests. The question requires mathematical modelling.

\section{Choosing and testing the model of} pulmonary dust retention

Although the physiological part played by the phagocytosis of dust particles deposited from the alveolar air is generally acknowledged, it has not been given a sufficiently good mathematical description. Most models for the kinetics of the elimination and retention of such particles and their translocation through lymphatics either do not describe their phagocytosis at all ${ }^{1-3}$ or do not attach sufficient importance to it. ${ }^{4}$ It is not surprising, then, that in the only paper devoted to differences in the retention of quartz and titanium dioxide, in which a method for modelling such differences was proposed, ${ }^{3}$ the authors used a formal (external) model of the process that does not describe the role of any of its mechanisms. Specifically it does not describe the phagocytosis of dust particles, nor the breakdown of lung macrophages and its consequences. Thus the authors did not use the most striking difference in biological effect between the compared materials, namely their essentially different cytotoxicity to macrophages, to explain the observed kinetic differences - for instance, the most characteristic: a considerably lower aptitude of titanium dioxide to translocation into 
tracheobronchial lymph nodes. The authors ensured good approximation to experimental data only by assuming, without any mechanistic arguments, that the transport of particles from the lung into lymph nodes begins only after the amount of dust retained in the lung parenchyma reaches a certain threshold. Moreover, in fitting the model to their data, they estimated this threshold to be considerably higher for titanium dioxide than for quartz.

This kind of approach would not provide an answer to the question formulated in the introduction to this paper. Therefore we use the model that was proposed previously for describing just kinetic processes directly or indirectly associated with the phagocytosis of particles and the resulting breakdown of lung macrophages. ${ }^{5}$

This model makes it possible to explicitly simulate differences due to unequal cytotoxicity of the dusts under comparison. It is also possible to check whether such simulation would be sufficient for predicting the accumulation of these dusts in the lung parenchyma and in tracheobronchial lymph nodes during chronic inhalation and after a long period of recovery after exposure. Thus the values should be close to corresponding experimental data.

Figure 1 shows the structure of the model. As the identification of its compartments and of the flows between them is clear from the figure and the arguments for this structure have been examined in detail previously in this journal, ${ }^{5}$ we do not dwell on them here. Briefly, the flows between the compartments are characterised by transfer rate constantsthat is, the proportion of the substance leaving the compartment per unit time in directions defined in the model-rather than by the mass of substance transported per unit time. In the notation adopted, $\mathrm{k}_{\mathrm{i}}$ is the constant of the rate of transfer from compartment $X_{i}$ to compartment $X_{i}$ or beyond the system modelled $\left(k_{\infty 7}\right)$. For long term experiments on animals, the week is the most convenient unit of time, so the dimension of the constants is weeks ${ }^{-1}$.

As this model considers only the fate of particles deposited in the pulmonary region, the rate of this deposition should be given. Taking into account the absence of both mathematical models for regional deposition of particles in the respiratory tract of rats and technically feasible methods of its direct measurement in small animals, we suggested ${ }^{5}$ an approach to its calculation from the data of a chronic experiment. Initially all the dust retained in the lung parenchyma is assumed to be contained in one compartment and the kinetics of its accumulation is described by the equation $y=a-b e^{-k t}$ the constants of which are selected so as to ensure satisfactory approximation to the experimentally determined mass of dust in the lung at various times of exposure. Assuming that this estimate of $k$ is the constant rate of dust output from the one compartmental model, the constant rate of input may be easily calculated. In going over to a multicompartmental model this constant is assumed to be the rate of the deposition of particles in the alveolar region $\bar{\omega}$. We have already explained why the mass of dust found in the lung after a sufficiently long exposure, a period of 24 hours after exposure or longer, and after bronchoalveolar lavage, may be assumed to be an experimental correlate of the sum of compartments $\left(\mathrm{X}_{4}\right.$ $\left.+\mathrm{X}_{6}\right)$ in such a multicompartment model. ${ }^{5}$ Thus the model describes the kinetics of interstitial pulmonary dust burden rather than that of total pulmonary burden, as it is based on experimental data which permit us only to estimate the first.

The same should be kept in mind when considering the results of the experiments described next.

Taking into account the limitations considered in the same paper, ${ }^{5}$ the set of constants
Figure 1 Structure of multicompartmental model for the kinetics of the retention and elimination of dust deposited in the alveolar region of the lung from Katsnelson et al. ${ }^{5}$

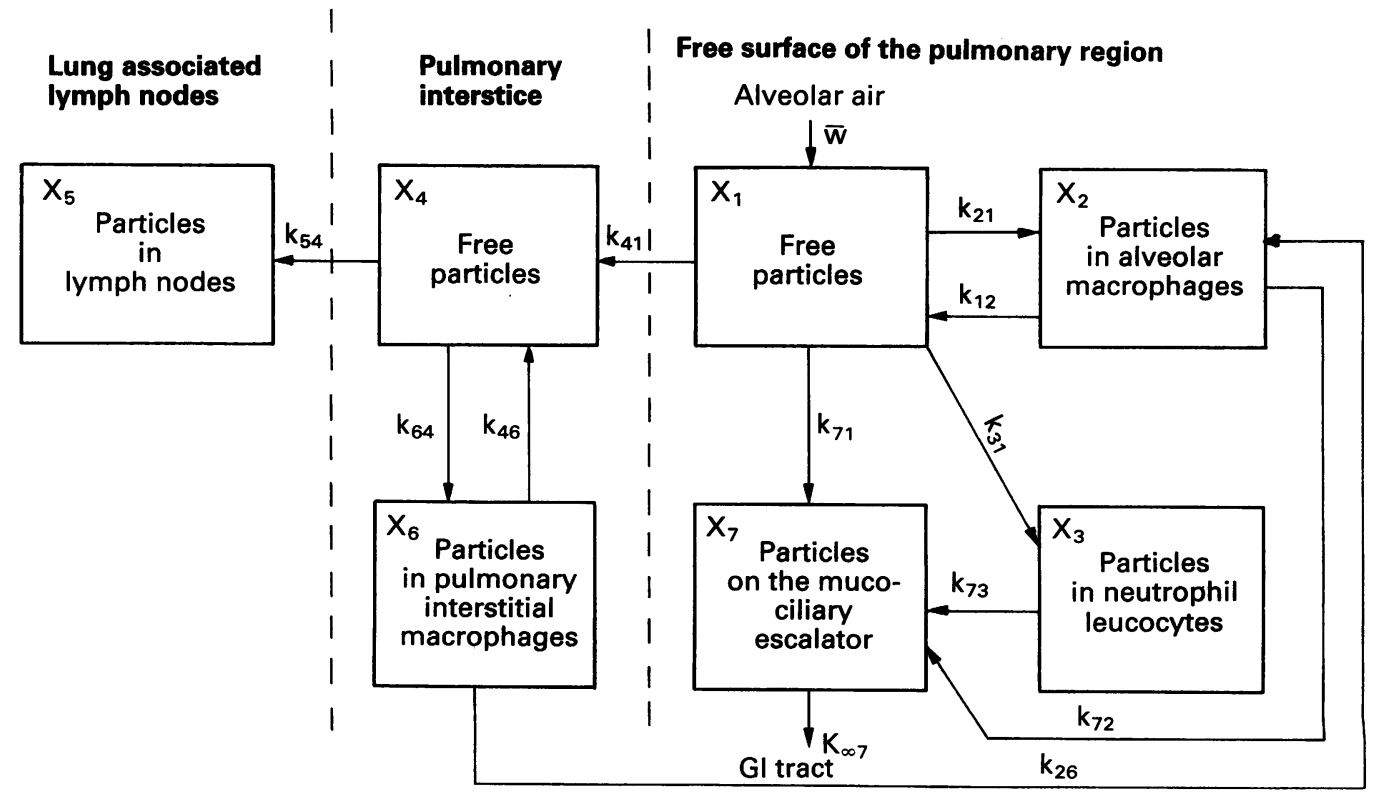


found with the help of a special computer program provided a satisfactory approximation to the amounts of quartzite dust found experimentally in the lung and in tracheobronchial lymph nodes during long term inhalation. ${ }^{5}$ This set required some adjustment after the first 20 weeks of exposure to describe the breakdown of the mechanism responsible for weakened recruitment of alveolar macrophages with its compensatory increase in neutrophilic leucocytes. The results of another such experiment required only minor adjustment of the constants from the same model. Before applying these constants to the problem of variations in cytotoxicity of dusts we checked once again if this model was adequate to describe the retention of quartzite dust from the same Pervouralsk mineral deposit.

The experiment was performed on randomly bred white female rats with an initial average body weight of $185 \mathrm{~g}$. The rats were exposed to the dusts in a whole body chamber for five hours a day, five times a week. Some of the animals were killed after the first 10 weeks, another group of rats was killed after 20 weeks of exposure, and another group at 10 weeks after the 20 week period of exposure. The average concentration of total airborne dust in the chamber as collected on special AFA aerosol plastic fibre filters (Isotop Co, Russia) without size separation over the total inhalation period was $81 \cdot 2(7 \cdot 9)$ $\mathrm{mg} / \mathrm{m}^{3}(\bar{x}(\mathrm{SEM}))$. The free silicon dioxide content of the lung and tracheobronchial lymph node tissue was measured after burning the samples. The ash was fused with a mixture of sodium bicarbonate and sodium chloride and then was leached in a $5 \%$ solution of sodium bicarbonate; after filtering and neutralising the solution with $0.5 \mathrm{M}$ sulphuric acid we measured the silicic acid by complex formation with ammonium molybdate in the presence of tartaric and acetylsalicylic acid and measurement of the complex spectrophotometrically at $600 \mathrm{~nm}$. Analyses at each experimental time point were performed on tissues from seven or eight rats from both the exposed and the control groups.

The constant of alveolar deposition $\bar{\omega}$ calculated by this method was $0.3 \mathrm{mg}$. week $^{-1}$, which is about a fifth of the value found in earlier experiments. This difference may be due partially to the use of another method for determining quartz in the tissue and partially to different particle sizes in the inhaled dusts. In any case, however, for a model describing the kinetics of subsequent dust retention and

Table 1 Kinetics of quartz retention in lung parenchyma and tracheobronchial lymph nodes (TBLN) during and after chronic inhalation exposure to dust and its simulation by means of a mathematical model

\begin{tabular}{|c|c|c|c|c|}
\hline \multirow[b]{2}{*}{ Duration of experiment } & \multicolumn{2}{|l|}{ Mass of dust in lungs ( $\mathrm{mg}$ ) } & \multirow{2}{*}{$\begin{array}{l}\text { Mass of dust } \\
\text { in TBLN } \\
(\mathrm{mg})^{*}\end{array}$} & \multirow[b]{2}{*}{$X_{5}$} \\
\hline & $\bar{x}(S E M)(95 \% C I)$ & $X_{4}+X_{6}$ & & \\
\hline $\begin{array}{l}10 \text { weeks of exposure } \\
20 \text { weeks of exposure } \\
10 \text { weeks after exposure }\end{array}$ & $\begin{array}{l}1.74(0.27)(1.09 \text { to } 2.39) \\
3.30(0.28)(2.57 \text { to } 4.03) \\
2.17(0.14)(1.81 \text { to } 2.53)\end{array}$ & $\begin{array}{l}1 \cdot 44 \\
2 \cdot 78 \\
2 \cdot 39\end{array}$ & $\begin{array}{l}0 \cdot 102 \\
0 \cdot 176 \\
0 \cdot 220\end{array}$ & $\begin{array}{l}0 \cdot 047 \\
0 \cdot 161 \\
0 \cdot 265\end{array}$ \\
\hline
\end{tabular}

^For the pooled tissue of the lymph nodes of all the rats in a given group in terms of one rat. elimination the variables of deposition should be given a priori. Therefore it is the reproducibility of the set of transfer rate constants rather than the absolute values that are of importance for checking the adequacy of such a model. To test this reproducibility we fixed all the values of the constants $k_{j i}$ that were found earlier in an experiment of the same duration, ${ }^{5}$ except the constant $k_{26}$, which was automatically determined by the computer such that the model would provide a satisfactory approximation to the data from our current experiment. The criterion of the best approximation (as built into the program) was minimisation of the sum of the squares of the deviations of experimental data from model predictions. If the best approximation found had been unsatisfactory, the next step would have been adjustment of some other constant, etc.

Table 1 shows that no such steps were necessary, because the model predictions fell within the $95 \%$ confidence intervals $(95 \%$ CIs) of all the experimental data on the lung and were close to the averaged data on tracheobronchial lymph nodes (with the exception of the first term of exposure). This degree of approximation may be regarded as sufficient for modelling chronic toxicokinetic processes. Also the model predicted: (1) a decrease in the rate of accumulation of quartz dust in the interstitium of the lungs during the second 10 weeks of exposure compared with the first 10 weeks (by $1.34 \mathrm{mg}$ compared with $1.44 \mathrm{mg}$ ). This agrees well with the experiment $(1.56 \mathrm{mg}$ compared with 1.74 $\mathrm{mg}$ ); (2) partial clearance of the lung parenchyma after exposure alongside a simultaneous increase in quartz transferred to tracheobronchial lymph nodes. Such lymphatic clearance, however, accounts for only a small proportion of the quartz dust eliminated from lungs over the 10 weeks after exposure. This fact confirms the essential role of the continuing elimination of particles from the interstitial lung tissue to the free surface in the pulmonary region and further through the tracheobronchial region towards the gastrointestinal tract. In the model, the part of this basic elimination route is played by the persisting flow from $\mathrm{X}_{6}$ into $\mathrm{X}_{2}$.

The most surprising thing, in our opinion,

Table 2 Constants of the seven compartment model describing the kinetics of quartz and titanium dust retention (week ${ }^{-1}$ )

\begin{tabular}{|c|c|c|c|}
\hline & \multicolumn{2}{|l|}{ Quartz } & \multirow[b]{2}{*}{$\begin{array}{l}\text { Titaniun } \\
\text { dioxide }\end{array}$} \\
\hline & $\begin{array}{l}\text { Experiment from } \\
\text { Katsnelson et al }\end{array}$ & $\begin{array}{l}\text { Current } \\
\text { experiment }\end{array}$ & \\
\hline$k_{12}$ & 0.05 & 0.05 & 0.016 \\
\hline $\mathbf{k}_{21}$ & 0.25 & 0.25 & 2.655 \\
\hline $\mathbf{k}_{26}$ & 0.06 & 0.05 & 0.215 \\
\hline $\mathbf{k}_{31}$ & 0.02 & 0.02 & 0.005 \\
\hline$k_{41}$ & 0.50 & 0.50 & 0.50 \\
\hline $\mathrm{k}_{46}$ & 0.03 & 0.03 & 0 \\
\hline$k_{54}$ & 0.01 & 0.01 & 0.01 \\
\hline$k_{64}$ & 0.15 & 0.15 & 1.316 \\
\hline $\mathbf{k}_{71}$ & $0 \cdot 10$ & $0 \cdot 10$ & $0 \cdot 10$ \\
\hline $\mathbf{k}_{72}$ & 0.05 & 0.05 & 0.05 \\
\hline $\mathrm{k}_{73}$ & $0 \cdot 10$ & $0 \cdot 10$ & $0 \cdot 10$ \\
\hline$k_{\infty 7}$ & 5.00 & 5.00 & $5 \cdot 00$ \\
\hline
\end{tabular}


is that all these results were obtained for a value of $k_{26}=0.053$, found by the computer, which is virtually equal to the previously reported value of $0 \cdot 06 .^{5}$ Table 2 shows the complete set of constants. Thus the model proposed earlier has been proved adequate once again.

\section{Modelling peculiarities of the kinetics of the retention and elimination of a poorly cytotoxic dust}

Most researchers regard titanium dioxide as a reference dust that is characterised by low fibrogenicity and low cytotoxicity. Although the often cited statement that this material is "biologically inert" does not stand serious criticism, its considerably lower cytotoxicity compared with quartz found support in a number of studies including ours. ${ }^{6}$ Therefore, we consider titanium dioxide to be suitable for modelling the peculiarities of the retention and elimination of a poorly cytotoxic dust. When modelling, we should be altering only those variables of the model that directly or indirectly depend on the intensity of the cytotoxic effect. They should be altered only in accordance with differences in cytotoxicity between titanium dioxide and the quartzite dust, the kinetics of which are described in the pulmonary region by the basic model.

The theoretical aim of such modelling as considered in the introduction to this paper does not require high accuracy of prediction. The modelling should, however, provide general agreement between predictions and experimental data if there is truth in the initial hypothesis about the key role of damage to lung macrophages and associated consequences in the kinetics of the retention of practically insoluble dust particles in the lung parenchyma and lymph nodes.

The experiment with titanium dioxide dust (rutile) was conducted in parallel to the experiment with quartzite dust for a similar average concentration of total dust in the chamber $\left(78 \cdot 8(11 \cdot 2) \mathrm{mg} / \mathrm{m}^{2}\right)$. The particle size distribution (as estimated by light microscopy of the dust samples with the same AFA filters made transparent by treatment with acetone vapours) was similar in both experiments. Thus the proportion of quartzite particles under $2 \mu \mathrm{m}$ was $59.0 \%$, and under $5 \mu \mathrm{m}$ was $92 \cdot 1 \%$; the proportion of particles of titanium dioxide under $2 \mu \mathrm{m}$ was $54.5 \%$ and under $5 \mu \mathrm{m}$ was $93.5 \%$. No particles over $20 \mu \mathrm{m}$ were discovered in the total count of about 500 particles in both cases.

Samples of dry lung and tracheobronchial lymph node tissues taken from rats killed at the same time points were burnt, fused with potassium pyrosulphate, and leached in hot $0.5 \%$ sulphuric acid. To the filtered solution was added a $10 \%$ solution of ammonia in the presence of ferric (III) sulphate and then a $5 \%$ solution of sulphuric acid until the red sediment disappeared. After the reduction of the iron (III) with a $3 \%$ solution of acetylsalicylic acid we added a $2 \%$ solution of bisodium salt of chromotropic acid to the sample and determined the concentration of titanium spectrophotometrically at $470 \mathrm{~nm}$. Each group had the same number of rats as in the experiment with quartzite dust.

For modelling we assumed the same value of $\bar{\omega}$ as it does not depend on cytotoxicity. For simulating the role of differences in cytotoxicity we changed the constants $k_{\mathrm{ii}}$ as follows: as the constant $k_{12}$ reflects the probability of breakdown of pulmonary alveolar macrophages whereas the constant $k_{46}$ reflects that of pulmonary interstitial macrophages, there is every reason to decrease both constants in the case of titanium dioxide compared with their values for quartzite. In our earlier studies we proved that the most informative criterion for the extent of breakdown of alveolar macrophages is the resulting recruitment of neutrophilic leucocytes. ${ }^{6}$ In chronic experiments, however, increased recruitment of neutrophils and an increase in their number in bronchoalveolar lavage (BAL) fluid may be due to other factors. $^{7}$ Therefore, comparison of the cellular composition of this fluid in rats that have inhaled titanium dioxide or quartzite for a long time reflects the comparative intensity of a chronic pathological process rather than the comparative cytotoxicity of different particles.

For estimating comparative cytotoxicity of different dusts we performed a special experiment with samples of these dusts taken in the exposure chambers. We injected $10 \mathrm{mg}$ of each dust intratracheally (as a sterile suspension in $1 \mathrm{ml}$ of normal saline) into rats in which BAL was performed once in six hours. The absolute number of neutrophilic leucocytes in rats that had received quartzite dust was $3.35(0.81) \times 10^{6} \quad\left(0.42(0.09) \times 10^{6}\right.$ in controls) whereas in those that had received titanium dioxide it was $0.85(0.16) \times 10^{6}$-that is, lower by a factor of 3.9. As in such tests this index is practically linearly dependent on the dose of macrophage breakdown products (administered into the lung or formed in it under the action of cytotoxic dust) it was possible to assume about a fourfold decrease in the constant $k_{12}$. Judging by the other criterion that we used for comparative estimation of the dust's ability to destroy alveolar macrophages, namely the neutrophilic leucocyte/alveolar macrophage ratio in BAL fluid $(6 \cdot 10(0.66)$ for quartzite and $1.99(0.65)$ for titanium dioxide) the reduction in this constant might be threefold. Thus in a model for the kinetics of titanium dioxide the values of the constant $\mathrm{k}_{12}$ may be assumed to be within the range of 0.0125 to 0.0164 weeks $^{-1}$.

Low probability of breakdown of alveolar macrophages in our model means a reduced number of particles penetrating into the lung interstitium (even with $k_{41}$ being unchanged). This suggests that the probability that titanium dioxide particles will act on interstitial macrophages is considerably lower than for quartz particles. In other words, the proportion of phagocytosing interstitial macrophages broken down as a result of this action is lower not only because of its specific intensity but also because of a lower dose of dust per pool 
Figure 2 Titanium dioxide content of rat lungs during and after chronic inhalation exposure; points $=$ experimental data; curve $=$ model prediction.

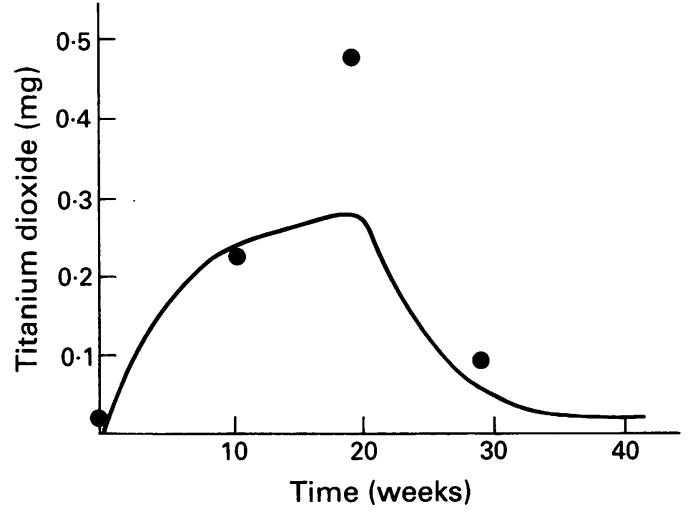

Figure 3 Titanium dioxide content of tracheobronchial lymph nodes of rats during and after chronic inhalation exposure; points = experimental data; curve $=$ model prediction.

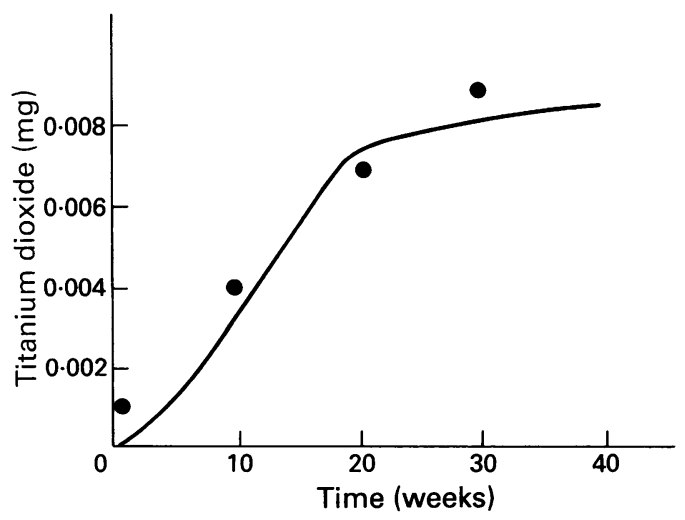

of interstitial macrophages available. Consequently, the constant $\mathrm{k}_{46}$ may be reduced for titanium dioxide compared with quartzite even more drastically than the constant $k_{12}$. The orders of magnitude thus obtained $\left(<0.001\right.$ week $\left.^{-1}\right)$ have a negligible effect on the mass of dust in the compartments as predicted by the model. The choice of a particular value of this order cannot be justified by the degree of approximation to experimental data and becomes uncertain. Therefore, $\mathrm{k}_{46}$ may be assumed to be equal to zero.

The probability that dust will be transported from the lung interstitium by recruited interstitial macrophages that turn into alveolar macrophages (from compartment $\mathrm{X}_{6}$ to compartment $\mathrm{X}_{2}$ in our model) depends on both the intensity of this recruitment and the number of particles in a single interstitial macrophage. Earlier we discussed the probability of interaction between interstitial macrophages and particles, but here we are interested in the probability that after such interaction interstitial macrophages would

Table 3 Kinetics of titanium dioxide retention in lung parenchyma and tracheobronchial lymph nodes during and after chronic inhalation exposure to dust and its simulation by means of a mathematical model

\begin{tabular}{lllll}
\hline & \multicolumn{2}{l}{ Mass of dust in lungs (mg) } & \multicolumn{2}{c}{$\begin{array}{c}\text { Mass of dust } \\
\text { in TBLN }\end{array}$} \\
\cline { 2 - 3 } Duration of experiment & $\bar{x}(S E M)(95 \%$ CI $)$ & $X_{4}+X_{6}$ & $(m g)^{*}$ & $X_{5}$ \\
\hline 10 weeks of exposure & $0.23(0.06)(0 \cdot 09-0.37)$ & 0.23 & 0.004 & 0.003 \\
20 weeks of exposure & $0.48(0 \cdot 15)(0 \cdot 12-0.84)$ & 0.28 & 0.007 & 0.007 \\
10 weeks after exposure & $0.09(0.07)(0-0.26)$ & 0.09 & 0.009 & 0.008 \\
\hline
\end{tabular}

ॠFootnote as for table 1 . retain viability and functional (phagocytic and migratory) activity. Judging by the data from studies on macrophage cultures and on cells in the BAL fluid both after intratracheal and chronic inhalation, macrophages never contain quartz particles in quantities that are uncountable. In the case of titanium dioxide, however, the percentage of such overloaded macrophages is high. The doses of dust being equal, this striking difference may be explained solely by the fact that the macrophage, even if it does not show obvious morphological and tinctorial signs of damage, sharply reduces or even completely loses its phagocytic ability after engulfing a few highly toxic particles, but retains phagocytic ability for a long time in the case of particles of low cytotoxicity. As in our model the constant $k_{26}$ means a proportion of particles inside the interstitial macrophages being transferred to $\mathrm{X}_{2}$ it should depend on the probability that a dust loaded macrophage migrating to the free surface of the acinus carries a high number of particles in it.

All these considerations suggest that the constant $\mathrm{k}_{26}$ should be increased rather than decreased when modelling the kinetics of titanium dioxide (which is in agreement with a sharp reduction in the constant $\mathrm{k}_{46}$ ). It was, however, impossible to even roughly estimate the factor of this increase. For the same reasons we had to increase the constants $k_{21}$ and $k_{64}$, which reflect the probability of a particle being phagocytosed by active alveolar and interstitial macrophages and thus how soon its activity is inhibited in the course of phagocytosis. It is also impossible to estimate the factor of the increase in these constants as it is impossible to count titanium dioxide particles inside individual macrophages. Finally, the lower the cytotoxicity of dust, the lower the total contribution of neutrophils to the alveolar phagocytosis of particles. ${ }^{6}$ This makes it possible to reduce the constant $k_{31}$ in proportion to the reduced recruitment of neutrophilic leucocytes at a single exposure to dust (that is: by a factor of 4 , and to assume it equals 0.005 ). Thus a particular value is chosen not by means of model approximation but is based on a priori considerations and independent experimental data. Therefore there is no need to assume it to be zero as in the case considered above.

Careful consideration of the model and its structure convinces us that with our assumptions we have exhausted all the changes in the values of the variables that may be justified by differences in the cytotoxicity of particles. Figures 2 and 3 and table 3 show the degree of approximation to experimental data that we consider sufficient. It is clear that predictions for compartment $\mathrm{X}_{5}$ agree or nearly agree with experimental data on the amount of titanium dioxide in tracheobronchial lymph nodes at all the four time points, but predictions for the sum of compartments $\left(\mathrm{X}_{4}+\mathrm{X}_{6}\right)$ agree with experimental data on the titanium oxide content of the lungs only at three of the four time points, namely at time zero, after the first 10 weeks of exposure, 
and 10 weeks after the 20 week exposure. For time zero, the experimental points in the plots (figs 2 and 3) represent the titanium dioxide contents of the lungs and tracheobronchial lymph nodes of control rats.

The overshoot of the experimental point corresponding to the amount of titanium dioxide in the lungs by the end of the 20 week exposure period may be due to a sampling error. This is indicated not only by the broadest confidence interval of the average result obtained at this time point (including the value predicted by the model) but also by the fact that the curve for accumulation and elimination drawn by the computer reflects the typical kinetics of the process in a more natural way, with gradual attainment of the retention plateau and sufficiently rapid but still gradual decay of the mass of dust in the lungs after exposure. If we assume that the experimental point under consideration as true, we have to conclude that the retention of titanium dioxide increased linearly over the entire 20 weeks without any tendency towards equilibrium (which is impossible) and dropped very steeply after the end of exposure-which has never been found for practically insoluble dusts.

Mention should be made, however, that in some inhalation experiments with titanium dioxide, the lung burden was also found to display a tendency towards a linear increase. ${ }^{8}$ Although such data cannot be disregarded, we still believe that, in the course of a long term experimental exposure of this kind, the tendency towards equilibrium would sooner or later establish itself. Otherwise the entire theory of pulmonary dust kinetics should be drastically reviewed. Indeed, why should comparatively harmless titanium dioxide particles impair the clearance mechanisms to a greater extent than very harmful quartz particles, for which the onset of equilibrium has been shown many times? The formation of a retention plateau depends on the relation between the rate of alveolar deposition and the efficiency of clearance. Experimenting with another harmful dust, the same researchers proposed that an impairment of the clearance mechanisms was responsible for the retention curve (lung burden) becoming linear. ${ }^{1}$

We think that for a model of this class, ideal approximation to seven experimental results out of eight may be considered satisfactory. Comparison of tables 1 and 3 shows that, in full compliance with the experiment, the model predicts lower retention of titanium dioxide than quartz, and an even greater difference in the retention of these dusts in tracheobronchial lymph nodes, and faster clearance of the lungs of titanium dioxide than of quartz.

Comparison of the sets of constants (table 2) shows that all these results were attained while adhering to the already formulated requirements of adaptation of the model. It was sufficient to take into account theoretically assumed kinetic consequences of reduced cytotoxicity for the mathematical description, of essential differences in the accumulation and elimination of differently cytotoxic insoluble particles.

\section{Predicting the intensity of pathological changes in lungs}

Earlier we proposed a toxicokinetic criterion, called the action integral, 5910 for predicting the intensity of pathological changes associated with the chronic retention of harmful substances in the organism (practically insoluble dust in the lungs in particular). The action integral is a definite integral of the function describing the accumulation of substances during chronic exposure and reduction in the accumulated mass as a result of stopping the exposure taken from the beginning of exposure to the moment at which the intensity of pathological changes due to these substances is estimated.

It is easy to see that the basic assumptions of this criterion are: (1) the harmful effect is linearly proportional to both the mass of retained substance and the time for which each discrete part of this mass stays in the organism (or a target organ); (2) equal duration of this time are toxicologically equivalent irrespective of the chronological part of the period within which they fall; (3) the reversal of pathological changes during the period after exposure can be neglected. Of course, the degree of validity of these assumptions is different for different pathological processes. Changes in the action integral associated with changes in the conditions of chronic inhalation of quartz for equal cumulative exposure ${ }^{9}$ or with changes in the kinetics of the retention of quartz in the lungs and the tracheobronchial lymphnodes due to a cytoprotector were shown to simulate quantitative changes in the intensity of silicosis in rats.

More recently other workers have suggested a basically similar (although mathematically different) criterion for predicting differences in the pathological response of the organism to quartz and titanium dioxide as measured by one index only, that is: the number of neutrophilic leucocytes in BAL fluid. ${ }^{3}$ As well as the kinetics of dust accumulation in the lungs these authors take into account a special "harmfulness function" as an additional factor when calculating their criterion, the incremental dose. This function describes the harmfulness of particles accumulated in the lung tissue. The concept of the action integral did not require such a factor as the aim was to predict the effect of exposure to the same substance under different conditions. As for predicting the effects produced by dusts of different cytotoxicity we decided to check whether the action integral needs the "harmfulness" of particles to be taken into account as well.

Having computed the values of the action integral for compartments $\mathrm{X}_{4}$ and $\mathrm{X}_{6}$ in our model, we found that the total action integral was $55.53 \mathrm{mg}$. week ${ }^{-1}$ for quartz, whereas for titanium dioxide it was only $5.57 \mathrm{mg}$. week $^{-1}$ -that is lower by a factor of 10 . The action 
integrals were computed for the entire 30 week experimental period. In this case we did not use the neutrophilic leucocyte count ratio that was used in short term tests on rats as the criterion of comparative "harmfulness" of these dusts. As was noted in the previous section of this paper, the neutrophilic leucocyte count corresponds to the number of destroyed alveolar macrophages. Also important are less noticeable cytotoxic effects produced by particles accumulated in the tissue. Therefore we directed our attention towards the comparative cytotoxicity of the dusts under study for a culture of rat peritoneal macrophages.

Judging by the trypane blue exclusion test, the percentage of non-viable macrophages in the control culture was $11 \cdot 78 \%(0 \cdot 14 \%)$, whereas in a culture incubated with a sample of quartzite dust from air from the exposure chamber it was $33.89 \%(0.78 \%)$ and in that with a similar sample of titanium dioxide dust $27 \cdot 80 \%(0 \cdot 44 \%)$. The difference between the exposed and control cultures for quartzite was a factor of 1.4 higher than that for titanium dioxide. For estimating the comparative cytotoxicity of a large number of dusts we also successfully used the 5'-nucleotidase activity reduction test as measured in $\mu \mathrm{g}$ of inorganic phosphorus. In this case it was equal to $18 \cdot 87 \%(0.65 \%)$ for the control culture and $9 \cdot 45 \%(1 \cdot 18 \%)$ and $13 \cdot 02 \%(0 \cdot 86 \%)$ for the cultures incubated with quartzite and titanium dioxide. The difference between the exposed and control cultures was 1.6 times higher for quartzite than titanium dioxide. Thus we assumed an average factor of 1.5 to express the higher aggressiveness of quartzite, and taking into account the 10-fold difference between the action integral values, quartzite may be expected to cause a pathological process in the lungs with an index of intensity 15 times greater than titanium dioxide.

If the difference in group average characteristics between the exposed and control groups is an index of the intensity of the pathological process, then the ratio of these indices for the dusts under comparison (table 4) shows that the prediction is true only for two criteria of intensity, the increase in the lipid content of lungs and the number of neutrophilic leucocytes in the BAL fluid. (We have already mentioned that against the background of a developed pneumoconiosis this number can be regarded not so much an index to the intensity of breakdown of alveolar macrophages at the moment of performing the BAL as a criterion for the intensity of the pathological process in the lungs.)

At the same time, the mass of the lungs and their hydroxyproline content are higher for quartzite than for titanium dioxide but only by a factor of three to five; however, 10 weeks after the exposure the difference in hydroxyproline content was already 10 -fold: $3270(155) \mu \mathrm{g}$ in the control; 5242(457) $\mu \mathrm{g}$ for quartzite (higher by $1972 \mu \mathrm{g}$ ); 3464(153) $\mu \mathrm{g}$ for titanium dioxide (higher by $194 \mu \mathrm{g}$ only). (Unfortunately we have no data on the retention of dust in the lung for this time point; therefore, we did not take it into account when checking the model.) Note also that at both time points the difference in the hydroxyproline content between lungs that accumulated low fibrogenic titanium dioxide and control lungs was not significant; therefore the true difference between this shift and the significant shift caused by quartzite could be higher.

The response of tracheobronchial lymph nodes, estimated in our experiments only by increase in their mass, provided experimental data given in table 4 that indicate that the shift due to quartzite is greater than that due to titanium dioxide by a factor of $9 \cdot 8$. Meanwhile, the value of the action integral for compartment $X_{5}$ is equal to 3.38 mg.week ${ }^{-1}$ in the case of quartzite and to $0 \cdot 15$ mg.week ${ }^{-1}$ in the case of titanium dioxidethat is, titanium dioxide is lower by a factor of $22 \cdot 5$. Taking into account the index of comparative "harmfulness" (that is: the factor of 1.5) a 34-fold difference between the effects can be predicted.

Predictions of this kind cannot be totally accurate quantitatively. We regard as chance the "accurate hit" for two indices of the five and regard the "deviation from the target" for the other three as normal. We think it more important that predictions based on the product of the action integral and the factor of comparative cytotoxicity are generally more reliable than predictions based on any of these individual criteria or on the estimate of the ultimate dust content of lungs only.

It is important that the experimental data and our predictions show that dusts of greater cytotoxicity cause a greater increasing dry mass of tracheobronchial lymph nodes than of the lungs. Indeed, quartzite caused a $9 \cdot 8$ times greater increase in the dry mass of tracheobronchial lymph nodes and only a $4 \cdot 65$ times greater increase in the lung mass compared with titanium dioxide, that is: compared with lungs the difference between dusts for tracheobronchial lymph nodes was higher

Table 4 Some indices of intensity of pathological changes in lungs of rats due to inhalation exposure to dusts at the end of a 10 week period after exposure (x SEM)

\begin{tabular}{|c|c|c|c|c|c|}
\hline $\begin{array}{l}\text { Groups of } \\
\text { rats }\end{array}$ & $\begin{array}{l}\text { Dry mass of } \\
\text { lungs (mg) }\end{array}$ & $\begin{array}{l}\text { Dry mass } \\
\text { of } T B L N(\mathrm{mg})\end{array}$ & $\begin{array}{l}\text { Hydroxyproline } \\
\text { in lungs, }(\mu g)\end{array}$ & $\begin{array}{l}\text { Lipids } \\
\text { in lungs (mg) }\end{array}$ & $\begin{array}{l}\text { Neutrophils } \times 10^{6} \\
\text { in BAL fluid }\end{array}$ \\
\hline $\begin{array}{l}\text { A Controls } \\
\text { B Exposed to quartz } \\
\text { C Exposed to titanum oxide }\end{array}$ & $\begin{array}{l}295 \cdot 2(8 \cdot 2) \\
486 \cdot 8(30 \cdot 05)^{\star} \\
336 \cdot 4(13 \cdot 2)^{\star} \dagger\end{array}$ & $\begin{array}{r}5 \cdot 5(0 \cdot 05) \\
23 \cdot 1(2 \cdot 9)^{\star} \\
7 \cdot 3(0 \cdot 5) \dagger\end{array}$ & $\begin{array}{l}2818(160) \\
3936(286)^{\star} \\
3257(232)\end{array}$ & $\begin{array}{c}29 \cdot 0(1 \cdot 4) \\
111 \cdot 7(13 \cdot 6)^{\star} \\
34 \cdot 9(2 \cdot 2) \dagger\end{array}$ & $\begin{array}{l}0.38(0 \cdot 09) \\
6 \cdot 21(0 \cdot 73)^{\star} \\
0 \cdot 77(0 \cdot 14)^{\star} \dagger\end{array}$ \\
\hline$\frac{B-A}{C-A}$ & $4 \cdot 65$ & $9 \cdot 78$ & $2 \cdot 55$ & $14 \cdot 02$ & 14.95 \\
\hline
\end{tabular}

${ }^{\star} \mathrm{p}<0.05 v$ controls irrespective of type of exposure.

$t \mathrm{p}<0.05$ groups exposed to titanium oxide $v$ corresponding group exposed to quartz. 
by a factor of $2 \cdot 1$. Our prognostic criterion obtained by multiplying the action integral by the comparative harmfulness index differs for these dusts for compartments $\left(\mathrm{X}_{4}+\mathrm{X}_{6}\right)$ by a factor of 15, whereas for compartment $X_{5}$ by a factor of 34 -that is, that difference is greater by a factor of $2 \cdot 3$. Agreement between the prediction and the experimental result is nearly perfect.

Another important consideration is that, according to most of the effects, the differences caused by the dusts in both lungs and tracheobronchial lymph nodes is more substantial than expected based only on the index of their comparative cytotoxicity without taking the action integral into account. Finally, the mass of the dusts under comparison in the interstitial lung 10 weeks after exposure differs greatly: by a factor of 24 in the experimental data, and by a factor of 27 in the model predictions (tables 1 and 3 ). Taking into account the 1.5 factor, this suggests about a 40 -fold difference in the intensity of pathological reaction if we took into account only the final retention of dust rather than the history of its accumulation and elimination as reflected by the action integral.

\section{Conclusion}

The cytotoxicity of dust particles has a dual role as a characteristic that determines the mathematical prediction of the comparative risk of developing pneumconiosis due to the action of different dusts. On the one hand, this characteristic determines the kinetics of the accumulation and retention of particles in lungs and lymph nodes, and thereby the value of the action integral. On the other hand, comparative cytotoxicity permits the comparative "harmfulness" of particles retained in the tissue of these organs to be judged.
Mathematical modelling shows that it is necessary to consider the combination of these two effects for explaining important quantitative differences between the pathological changes due to chronic inhalation of practically insoluble dusts of different cytotoxicity, assuming similar conditions for their deposition from the alveolar air.

1 Bolton RE, Vincent JH, Jones $\mathrm{AD}$, Addison JA, Beckett ST. An overload hypothesis for pulmonary clearance of UICC amosite fibers inhaled by rats. Br $f$ Ind Med 1983;40:264-72.

2 Vincent JH, Johnston AM, Jones AD, Bolton RE, Addison JA. Kinetics of deposition and clearance of inhaled minJA. Kinetics of deposition and clearance of inhaled mineral dusts during

3 Vincent $\mathrm{JH}$, Donaldson K. A dosimetric approach for relating the biological response of the lung to the accumulation of inhaled mineral dust. $\mathrm{Br} \mathcal{F}$ Ind $\mathrm{Med}$ 1990;47:302-7.

4 Smith TJ. Development and application of a model for estimating alveolar and interstitial dust levels. $A n n$ Occup Hyg 1985;29:495-516.

5 Katsnelson BA, Konysheva LK, Privalova LI, Morozova KI. Development of a multicompartmental model of the $\mathrm{K}$. Dev of pulmonary region of the lung during chronit

6 Katsnelson BA, Privalova LI. Recruitment of phagocytizing cells into the respiratory tract as a response to the cytotoxic action of deposited particles. Environ Health

7 Privalova LI, Katsnelson BA, Yelnichnykh LN. Some peculiarities of the pulmonary phagocytotic response: dust retention kinetics and silicosis development durin long-term exposure of rats to high quartz dust levels. $B$ f Ind Med 1987;44:228-35.

8 Vincent $\mathrm{JH}$, Jones $\mathrm{AD}$, Johnston AM, McMillan C Bolton RE, Cowie H. Accumulation of inhaled minera dust in the lung and associated lymph nodes: implications for exposure and dose in occupational lung disease. Ann Occup Hyg 1987;31:375-93.

9 Baidosov VA, Katsnelson BA, Privalova LI. An approach to the VA, Katherach organization mulative working period unde exposure to harmful substances with marked cumulative ability, taking pulmonary dust retention as example. In Mazurov I D, Smirnov A I, eds. Mathematical models of biological and medical systems. Sverdlovsk, Russia Publishing House Nauka, 1988:67-8 (in Russian)

10 Privalova LI, Katsnelson BA, Baidosov VA. The "action integral" as a criterion for comparative assessment and control of exposure patterns to substances with marked material cumulation. Gig Sanit 1986;12:12-5 (in Russian) 